\title{
PREPRINT
}

\section{Age-related decline of cognitive resources precedes and explains the decline in physical activity}

\author{
Boris Cheval ${ }^{1,2^{*}}$, Dan Orsholits ${ }^{3}$, Stefan $_{\text {Sieber }}^{3}$, Delphine Courvoisier ${ }^{3,4}$, Stéphane Cullati ${ }^{3,4}$, \\ Matthieu P. Boisgontier ${ }^{3,5,6^{*}}$
}

${ }^{1}$ Swiss Center for Affective Sciences, University of Geneva, Switzerland

${ }^{2}$ Laboratory for the Study of Emotion Elicitation and Expression (E3Lab), Department of Psychology, University of Geneva, Switzerland

${ }^{3}$ Swiss NCCR “LIVES - Overcoming Vulnerability: Life Course Perspectives", University of Geneva, Switzerland

${ }^{4}$ Department of General Internal Medicine, Rehabilitation and Geriatrics, University of Geneva, Switzerland

${ }^{5}$ Department of Physical Therapy, University of British Columbia, Vancouver, Canada

${ }^{6}$ Department of Movement Sciences, KU Leuven, Belgium

*Corresponding authors: Campus, Biotech, Chemin des mines 9, 1202 Genève, Switzerland; boris.cheval@unige.ch; @ChevalBoris (B. Cheval). Faculty of Medicine, 212-2177 Wesbrook Mall, Vancouver BC V6T 1Z3, Canada; matthieu.boisgontier@ubc.ca; @MattBoisgontier (M.P. Boisgontier).

Citation: Cheval B, Orsholits, D, Sieber, Courvoisier D, Cullati, Boisgontier MP (2019) Agerelated decline of cognitive resources precedes and explains the decline in physical activity. SportRxiv. DOI: https://doi.org/10.31236/osf.io/pagx6

\begin{abstract}
Objective: This study aimed to test whether the level of cognitive resources explain the engagement in physical activity across aging and whether the age-related decline of cognitive resources precede the decline in physical activity. Methods: Data from 105,206 adults aged 50 to 90 years from the Survey of Health, Ageing and Retirement in Europe (SHARE) were used in adjusted linear mixed models to examine whether the engagement in moderate physical activity and its evolution across aging was dependent on cognitive resources. Cognitive resources and physical activity were measured 5 times over a 12-year period. Delayed recall, verbal fluency, and the level of education were used as indicators of cognitive resources. The frequency of engagement in moderate physical activity was self-reported. Dynamic structural equation models (SEM) were used to assess the temporal precedence of changes in cognitive resources and physical activity. Results: Results showed that lower cognitive resources were associated with lower levels and steeper decreases in moderate physical activity across aging. Results further revealed a time-ordered effect with a stronger influence of cognitive resources (delayed recall and verbal fluency) on subsequent changes in moderate physical activity than the opposite. Conclusion: These findings suggest that, after age 50, the level of engagement in moderate physical activity and its trajectory depend on the availability of cognitive resources.
\end{abstract}

Keywords: physical activity; aging: cognition; bidirectional associations 


\section{Introduction}

In healthy aging, observational studies have widely reported the protective effect of physical activity on cognitive functioning. ${ }^{1-6}$ Yet, the evidence stemming from intervention studies in older adults is inconclusive. Some studies observed this protective effect of physical activity, ${ }^{7}$ 8 whereas more recent ones did not. ${ }^{9-13}$ Other studies supported the prospective effect of cognition and education on physical activity. ${ }^{14}{ }^{15}$ Finally, one study suggested that the effect of cognitive functioning on physical activity was stronger than the effect in the opposite direction. ${ }^{16}$ Taken together, these findings question the unidirectionality of the relationship between cognition and physical activity. To the best of our knowledge, large-scale longitudinal studies have never been used to clarify the nature of this relationship.

To investigate whether cognitive resources explain the frequency of engagement in moderate physical activity and their trajectories across aging, we used data from 105,206 adults aged 50 years and older collected over 12 years. Both the effect of inter-individual differences and intraindividual changes in cognitive resources were tested. Based on a recent theory contending that cognitive resources are required to counteract the attraction to energetic cost minimization, ${ }^{17,41}$ thereby facilitating the engagement in physical activity, we hypothesized that lower cognitive resources (both inter- and intra-individual) are associated with less frequent engagement in physical activity (H1) and with a more pronounced decline of this engagement across aging (H2). We expected the decrease in cognitive resources to precede the decrease in physical activity (H3).

\section{Methods}

\section{Population and design}

Our analyses used data from the Survey of Health, Ageing and Retirement in Europe (SHARE) ${ }^{17}$ a European database of individuals aged 50 or older including 5 repeated measurements between 2004 and 2015. Physical activity and cognitive function (delayed recall and verbal fluency) were assessed at measurement 1, 2, 4, 5, and 6. Education was measured when the participant joined the study. Participants aged 50 to 90 years with at least one measure of physical activity and cognitive functioning were included in our analysis. The relevant ethics committees in the participating countries approved SHARE. All participants provided written informed consent.

\section{Measures}

Physical activity. Moderate physical activity was measured using the following item: ${ }^{15}$ 1819 "How often do you engage in activities that require a low or moderate level of energy such as gardening, cleaning the car, or doing a walk?" Participants answered on a 4-point scale: 1, more than once a week; 2 , once a week; 3 , one to three times a month; 4 , hardly ever, or never. In the models, the variable was reversed so that higher values indicated higher physical activity.

Cognitive resources. Cognitive resources were measured using the following three indicators: delayed recall, verbal fluency, and highest educational attainment. In the 10-word delayed recall test, ${ }^{20}$ participants listened to a list of ten words that were read out loud by the interviewer. Immediately after reading the wordlist, the participants were asked to recall as many words as possible. This was asked again after a delay during which the verbal fluency took place. The latter delayed recall score is the number of words that the respondent is able to recall, which ranges from 0 to 10 . In the verbal fluency test, ${ }^{21}$ participants named as many different animals as they could think of in 60 seconds. The score consisted of the total number of correctly named animals. Verbal fluency and delayed recall tasks were used to assess fluid cognitive abilities, ${ }^{22}$ with verbal fluency reflecting executive functioning (e.g., as executive 
control, selective attention or selective inhibition $)^{23}$ and delayed recall reflecting earlycognitive impairment. ${ }^{24}$ Yet, it is important to acknowledge that emerging research suggests that verbal fluency is also highly related to verbal skills and thus, is not a perfect measure of executive functioning. ${ }^{25}$ UNESCO's International Standard Classification of Education (ISCED) was used to group participants into primary, secondary, and tertiary levels of education. ${ }^{26}$ This latter measure was used to assess cognitive reserve. ${ }^{27}$

Covariates. The following covariates were used: gender, measurement occasions, birth cohort, attrition, chronic health conditions, country of residence, and dementia (Supplemental Material 1).

\section{Statistical analysis}

Linear mixed models were used to examine the effect of cognitive resources on engagement in moderate physical activity and its trajectory across aging. By accounting for the nested structure of the data (i.e., repeated observations within a single participant) ${ }^{28}$ these models allow examining both the average engagement in physical activity across aging and the interindividual variabilities in these levels as well as the rate of change. These models can separate within- and between-person effects by introducing both the individual mean value of a particular variable and the deviation from this mean at each time point. The coefficient of the mean value estimates inter-individual differences. The coefficient of the deviation estimates intra-individual changes.

The fitted models included linear age, quadratic age, and the covariates as fixed effects. Their random structure encompassed random intercepts for participants and random linear slopes for the repeated measurements at the level of participants. These random effects estimated each participant's engagement in physical activity and the rate of change of this engagement over time. Age was centred at the midpoint of the sample's age range ( 70 years) and was then divided by 10 . Thus, a 1-unit change in the coefficients yielded effects on the physical activity rate of change over a 10-year period. Model 1 tested the association of an indicator of cognitive resources (within- and between-person effects) and the mean level of physical activity in older adults. In model 2, interaction terms between cognitive resources (within- and between-person effects) and linear and quadratic age were included to assess the influence of cognitive resources on physical activity trajectories. The quadratic effect of age was added to account for the potential influence of cognitive resources on the accelerated (or decelerated) decline of physical activity across aging. Specifically, the interactions of between-participant differences with age tested whether between-participant differences in cognitive resources moderated the effect of age. For example, individuals with lower cognitive resources may show a faster decline of physical activity across aging. The interactions of within-person differences with age tested whether having a lower (vs. higher) level of cognitive resources than usual influenced physical activity trajectories. For instance, a faster decline in physical activity may be observed in individuals with a lower level of cognitive resources than usual. The models were fitted with one indicator of cognitive resources at a time. We also fitted a fully-adjusted model including the three indicators of cognitive resources. Statistical analyses were performed using the lme4 and lmerTest $\mathrm{R}$ packages. ${ }^{29-31}$ Pseudo $\mathrm{R}^{2}$ were calculated to estimate effect size.

We performed four sensitivity analyses: 1) including health behaviours (smoking, alcohol consumption, dietary behaviour), health-related covariates (depression, body mass index, and self-rated health), and additional sociodemographic variables (partner status and satisfaction with household income); 2) excluding participants with dementia; 3) excluding participants 
who died during the survey; 4) excluding participants who dropped out during the survey. Supplemental Material 2 provides more details on all covariates used in the sensitivity analyses.

Bivariate latent change score models (BLCSMs) ${ }^{32}$ were used to examine the temporal precedence of changes in cognitive resources and physical activity. BLCSMs are structural equation models (SEM) that combine latent growth curves models ${ }^{33}$ with autoregressive crosslag models. ${ }^{34}$ We adopted a step-by-step modelling strategy. ${ }^{35-37}$ The fitted models included dynamic parameters: coupling and auto-proportional effects. BLCSM1 did not include crosslag paths between cognitive resources and physical activity and served as the baseline comparison model. In BLCSM2, a unidirectional coupling from cognitive resources predicting subsequent changes in physical activity was included. In BLCSM3, a unidirectional coupling from physical activity to predicting subsequent changes in cognitive resources was included to BLCSM1. BLCSM4 was a bidirectional coupling model included both pathways. BLCSM2 assessed the temporal precedence of cognitive resources on physical activity, BLCSM3 the precedence temporal of physical activity on cognitive resources, and BLCSM4 the reciprocal associations between cognitive resources and physical activity. The models were run separately for each cognitive indicator (verbal fluency and delayed recall). The models were fitted using Mplus $^{38}$ with full-information maximum-likelihood estimation and were tested against the baseline model (BLCSM1). A likelihood ratios test of changes was performed to determine whether the coupling parameters were significant. The alpha criterion was set to .01. The weighted Bayesian information criterion $[\mathrm{w}(\mathrm{BIC})]^{39}$ was used to select the best fitting model. All the models were also tested using vigorous physical activity (Supplemental Material 3).

\section{Results}

At baseline, $11.4 \%$ of the participants were hardly ever or never active, $6.0 \%$ were active 1 to 3 times per month, $13.8 \%$ were active once a week, and $68.7 \%$ were active more than once a week (Table 1). Physically active participants showed better delayed recall and verbal fluency, higher education, lower dementia, and were less likely to be older, to be a woman, and to drop out or die during the survey than physically inactive participants (Table 1).

\section{Cognitive resources and level of physical activity.}

Results showed that lower levels of delayed recall, verbal fluency, and education were associated with lower engagement in physical activity (Table 2 and Table S1). For delayed recall and verbal fluency, this association was observed at the between- and within-person level. At the between-person level, participants with lower levels of delayed recall or verbal fluency showed lower engagement in moderate physical activity. At the within-person level, lower delayed recall or verbal fluency were associated with lower engagement in moderate physical activity. These associations remained significant in the fully-adjusted model. Results of the sensitivity analyses were consistent with the main analyses (Table S5).

\section{Cognitive resources and physical activity trajectories.}

Results showed that lower levels of delayed recall, verbal fluency, and education were associated with a steeper decline of physical activity across aging (Figure 1; Table 2 and Table S1). At the between-person level, participants with lower levels of delayed recall, verbal fluency, and education showed an accelerated decline of physical activity across aging. In other words, the influence of cognitive resources on the engagement in moderate physical activity was more pronounced as adults grew older. At the within-person level, decreases in delayed recall or verbal fluency were associated with a faster decline of physical activity, but without acceleration across aging. In the fully-adjusted model, these associations were attenuated but cognitive resources remained associated with physical activity trajectories. In the latter model, 
cognitive resources explained $5.8 \%$ and $6.4 \%$ of the inter-individual variance of the level and trajectories of moderate physical activity, respectively. Taken together, all the variables explained $21.8 \%$ and $19.2 \%$ of the inter-individual variance of the level and trajectories of moderate physical activity, respectively. Results of the sensitivity analyses were consistent with the main analyses (Table S5).
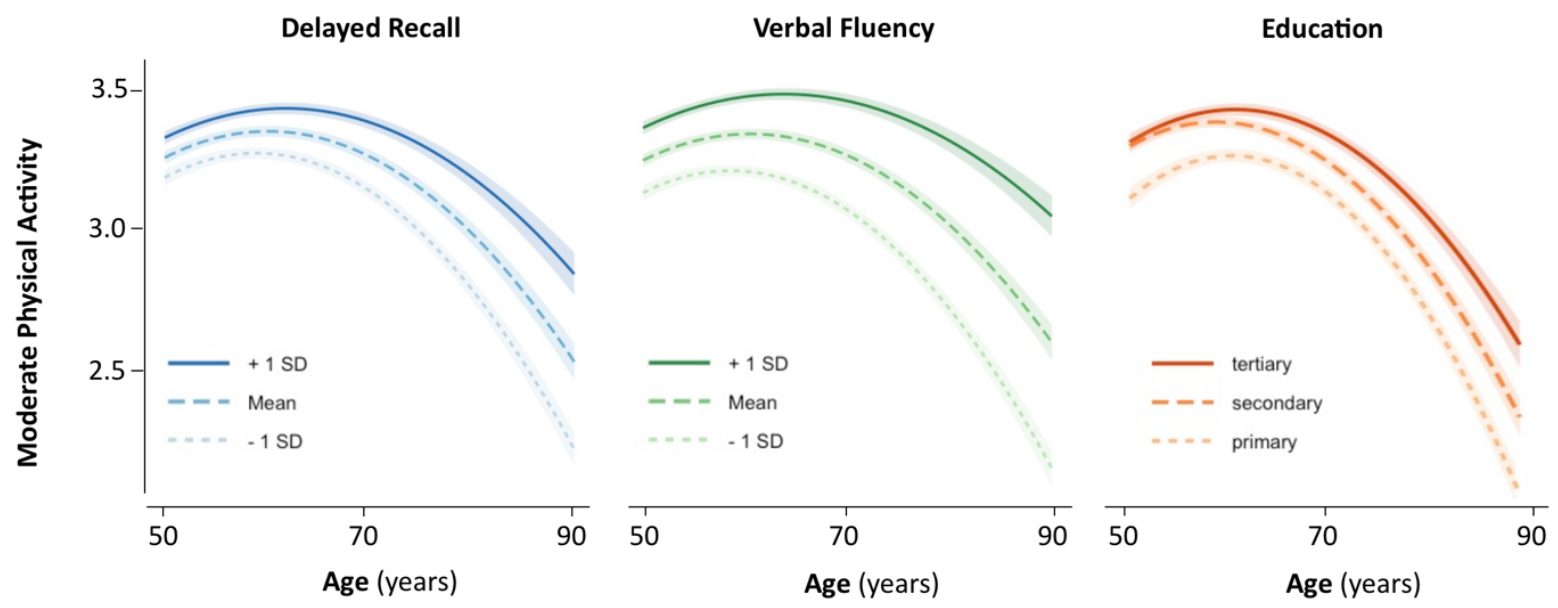

Figure 1. Associations of cognitive resources with the trajectories of moderate physical activity across aging. For delayed recall and verbal fluency, the variables were standardized. The coefficients are interpreted as the effect of an increase of one standard deviation.

\section{Temporal precedence.}

Results showed a fit improvement when the cognitive resources (delayed recall and verbal fluency) $\rightarrow$ change in moderate physical activity coupling was included in the baseline model (BLCSM2 vs. BLCSM1). Including the moderate physical activity $\rightarrow$ change in cognitive resources coupling also improved the fit (BLCSM3 vs. BLCSM1), but to a lesser extent. The bidirectional couplings model showed better fit than the baseline model (BLCSM4 vs. BLCSM1). This better fit was mainly explained by the cognitive resources $\rightarrow$ change in moderate physical activity coupling. The weighted Bayesian information criterion favored BLCSM2 over all the other models. BLCSM2 accounted for $81.5 \%$ (for delayed recall) and $72.8 \%$ (for verbal fluency) of relative predictive accuracy. BLCSM3 accounted for $9.5 \%$ (for delayed recall) and 3.3\% (for verbal fluency) of predictive accuracy. Overall, results of this step-by-step modelling strategy clearly showed that the model including the effect of the cognitive resources $\rightarrow$ changes in physical activity coupling (BLCSM2) was the model that best fitted the data (Table 3; Figure 2). 


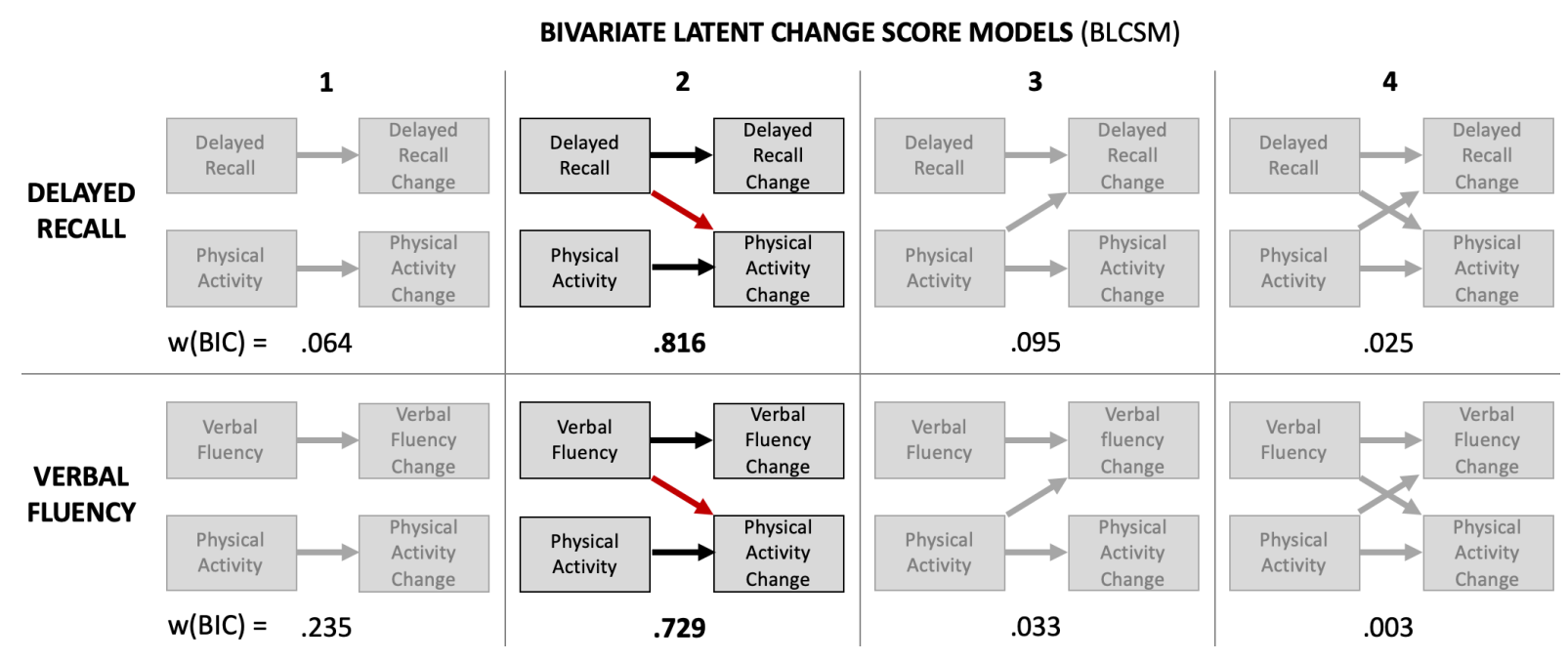

Figure 2. Fit of the bivariate latent change score models (BLCSM1 to 4) in two cognitive resources that change across aging (delayed recall and verbal fluency).

BLCSM1 = baseline comparison model. BLCSM2 = unidirectional coupling from cognitive resources predicting subsequent changes in physical activity. BLCSM3 = unidirectional coupling from physical activity to predicting subsequent changes in cognitive resources. BLCSM4 = bidirectional coupling model included both pathways. $\mathrm{w}(\mathrm{BIC})=$ weighted Bayesian information criterion. BLCSM2 best fitted the data in both cognitive resources.

\section{Discussion}

\section{Main findings.}

This study aimed to test whether cognitive resources were associated with the frequency of engagement in physical activity and its evolution across aging. In this large-scale longitudinal study including 21 European countries and 105,206 older adults, results showed that lower levels of cognitive resources were associated with less frequent engagement in moderate physical activity and with a steeper decline of this engagement across aging. These effects were observed across three cognitive indicators (delayed recall, verbal fluency, and education) and two levels of analysis (within- and between-person). These associations remained significant after adjusting for health behaviours, health-related covariates, and sociodemographic variables. Results further revealed, for the first time, that the decline of cognitive resources preceded the decline of moderate physical activity, with a weaker association in the opposite direction. Taken together, these results demonstrate that the engagement in moderate physical activity and its trajectory after age 50 depends on cognitive resources.

\section{Comparison with previous studies.}

Results showed that lower cognitive resources were associated with lower engagement in moderate physical activity in adults aged 50 years and older (H1). This association was consistent across the three indicators of cognitive resources at the within- and between-person levels. These findings support previous studies that investigated the association between cognitive functioning and physical activity. ${ }^{1-5}$

Results showed that lower levels of cognitive resources were associated with an accelerated decline of physical activity at the within- and between-person levels (H2). This association was robust after adjusting for multiple sociodemographic and health-related covariates, thereby confirming the critical role of cognitive resources in explaining physical activity. Of note, the results were consistent across the three indicators of cognitive resources, which targeted 
somewhat different cognitive processes. Therefore, multiple dimensions of cognitive resources may be required to engage in physical activity.

To the best of our knowledge, this study is the first to formally test the temporal precedence of cognitive resources and moderate physical activity. Results showed that the influence of cognitive resources on subsequent changes in physical activity was stronger than the influence in the opposite direction (H3). While previous studies focused on the protective effect of physical activity on cognitive functioning ${ }^{7}{ }^{8}$, our results showed that this relationship is not unidirectional. Cognitive resources are required to engage in physical activity and to slow down the decline of this engagement over the years. A potential explanation for these time-ordered associations is that cognitive resources are critical in counteracting a general tendency to minimize energetic expenditure and to engage in physical activity. ${ }^{40-42}$ This explanation is supported by recent results showing that avoiding sedentary behavior opportunities are associated with higher brain activity in the frontal lobe. ${ }^{41}$ The availability of cognitive resources supporting the engagement in physical activity may be particularly important in modern societies, where opportunities to adopt sedentary behaviors are ubiquitous. ${ }^{43}$ The fact that physical activity was also associated, although to a weaker extent, with subsequent changes in cognitive resources suggested a reciprocal relationship. This result is consistent with previous studies suggesting that physical activity is a determinant of cognitive functioning, ${ }^{4} 77$ although most recent studies and those with a long follow-up (i.e., less prone to reverse causation biases) found no evidence of a causal effect of physical activity on cognitive decline. ${ }^{9-12} 14$

\section{Strengths and weaknesses.}

This study has many strengths. First, the repeated measurement of physical activity allowed investigating its evolution over 40 years (i.e., from age 50 to 90). Second, the repeated assessment of cognitive resources allowed examining the influence of inter-individual differences and intra-individual changes. Third, the inclusion of more than 105,000 participants living in 21 European countries provided reliable and generalizable results. Fourth, sensitivity analyses excluding participants with dementia controlled for the potential confounding influence of this cognitive impairment. Fifth, results were robust after adjusting for health behaviours, health-related covariates, and additional sociodemographic variables. Sixth, this study was the first to formally test the temporal precedence of changes in cognitive resources and physical activity using dynamic structural equation models.

However, potential limitations should also be noted. The first limitation is related to physical activity. Our results were based on self-reported measures of moderate physical activity, which creates the potential for misclassification bias. ${ }^{44}$ However, the potential inaccuracy of these self-reports is unlikely to explain the observed associations between cognitive resources and physical activity. Moreover, the scale lacked granularity, which prevented the assessment of specific physical activity levels that are associated with health benefits, such as the 30 minutes of moderate physical activity intensity 5 times per week. Studies accurately determining thresholds of physical activity are needed. Additionally, the examples of physical activities used in the scale (e.g., gardening, cleaning the car) may have biased participants' response toward activities that were more specifically associated with these social contexts. It should also be noted that the results of the models examining vigorous physical activity showed a lower impact of cognitive resources on physical activity as adults grew older (Supplemental Material 2). Results of the cross-lagged dynamic structural equation models failed to converge. These results suggest that the processes reported in here are specific to moderate physical activity. The processes linking cognitive resources and vigorous physical activity may be different. The second limitations are potential selection biases related to the SHARE design. The recruitment 
procedure occurred late in life (i.e., after 50 years old) and participants who were able to respond to SHARE may have specific characteristics. The second selection bias was due to the loss of participants during the follow-up, which cannot be excluded, like in all long-term prospective studies. To attenuate this bias, our statistical analyses were adjusted for attrition and sensitivity analyses excluded participants who died or dropped out during the follow-up. The third limitation is related to the cognitive resources. Verbal fluency was used to assess fluid cognitive abilities, specifically executive control and inhibiting functions. ${ }^{23} 45$ Yet, previous studies showed that verbal fluency was not only affected by executive control ability, but also by verbal ability. ${ }^{25}$ Therefore, future studies could use tasks that are specifically designed to tap into the different domains of executive functions to determine their relative contribution to physical activity. ${ }^{46}$

\section{Conclusion and policy implications.}

Lower cognitive resources are robustly associated with lower engagement in moderate physical activity and faster decline of this engagement across aging. These findings support recent studies suggesting that cognitive resources are required to counteract a general attraction to effort minimization. Interventions aiming to prevent the decline of cognitive resources should be implemented to improve the engagement in physical activity, which is associated with extensive health benefits. ${ }^{47,48}$

Author contributions: B.C. and M.P.B. designed the analyses. B.C. and M.P.B. analysed the data. B.C. and M.P.B. drafted the manuscript. All authors critically appraised and approved the final version of the manuscript.

Funding: B.C. is supported by an Ambizione grant (PZ00P1_180040) from the Swiss National Science Foundation (SNSF).

Conflict of interest: The authors declare no conflict of interests.

Acknowledgements: The authors would like to thank Stephen Aichele for the support on the bivariate latent change score models. This work uses data from SHARE Waves 1, 2, 3 (SHARELIFE), 4, 5 and 6 (DOIs: 10.6103/SHARE.w1.600, 10.6103/SHARE.w2.600, 10.6103/SHARE.w3.600, 10.6103/SHARE.w4.600, 10.6103/SHARE.w5.600, 10.6103/SHARE.w6.600), The SHARE data collection was primarily funded by the European Commission through FP5 (QLK6-CT-2001-00360), FP6 (SHARE-I3: RII-CT-2006-062193, COMPARE: CIT5-CT-2005-028857, SHARELIFE: CIT4-CT-2006-028812) and FP7 (SHARE-PREP: no.211909, SHARE-LEAP: no.227822, SHARE M4: no.261982). Additional funding from the German Ministry of Education and Research, the Max Planck Society for the Advancement of Science, the U.S. National Institute on Aging (U01_AG09740-13S2, P01_AG005842, P01_AG08291, P30_AG12815, R21_AG025169, Y Y1-AG-4553-01, IAG_BSR06-11, OGHĀ_04-064, HHSN271201300071C) and from various national funding sources is gratefully acknowledged (see http://www.share-project.org).

Inform consent: Informed consent was obtained from all participants included in the study.

Ethical approval: This study was part of the SHARE study, approved by the relevant research ethics committees in the participating countries, and all participants provided written informed consent. 
Data sharing: This SHARE dataset is available at http://www.share-project.org/dataaccess.html.

\section{References}

1. Baumgart M, Snyder HM, Carrillo MC, et al. Summary of the evidence on modifiable risk factors for cognitive decline and dementia: a population-based perspective. Alzheimer Dement 2015;11(6):718-26.

2. Sofi F, Valecchi D, Bacci D, et al. Physical activity and risk of cognitive decline: a metaanalysis of prospective studies. J Intern Med 2011;269(1):107-17.

3. Morgan GS, Gallacher J, Bayer A, et al. Physical activity in middle-age and dementia in later life: findings from a prospective cohort of men in Caerphilly, South Wales and a metaanalysis. J Alzheimers Dis 2012;31(3):569-80.

4. Blondell SJ, Hammersley-Mather R, Veerman JL. Does physical activity prevent cognitive decline and dementia?: A systematic review and meta-analysis of longitudinal studies. BMC public health 2014;14(1):510.

5. Hamer M, Terrera GM, Demakakos P. Physical activity and trajectories in cognitive function: English Longitudinal Study of Ageing. J Epidemiol Community Health 2018;72(6):47783.

6. Lindwall M, Cimino CR, Gibbons LE, et al. Dynamic associations of change in physical activity and change in cognitive function: Coordinated analyses of four longitudinal studies. J Aging Res 2012;2012:793598.

7. Colcombe S, Kramer AF. Fitness effects on the cognitive function of older adults: a metaanalytic study. Psychol Sci 2003;14(2):125-30.

8. Angevaren M, Aufdemkampe G, Verhaar H, et al. Physical activity and enhanced fitness to improve cognitive function in older people without known cognitive impairment. Cochrane Database Syst Rev 2008;3(3):1-73.

9. Young J, Angevaren M, Rusted J, et al. Aerobic exercise to improve cognitive function in older people without known cognitive impairment. Cochrane Database Syst Rev 2015;4:CD005381.

10. Snowden M, Steinman L, Mochan K, et al. Effect of exercise on cognitive performance in community-dwelling older adults: review of intervention trials and recommendations for public health practice and research. J Am Geriatr Soc 2011;59(4):704-16.

11. Andrieu S, Guyonnet S, Coley N, et al. Effect of long-term omega 3 polyunsaturated fatty acid supplementation with or without multidomain intervention on cognitive function in elderly adults with memory complaints (MAPT): a randomised, placebo-controlled trial. Lancet Neurol 2017;16(5):377-89.

12. Sink KM, Espeland MA, Castro CM, et al. Effect of a 24-month physical activity intervention vs health education on cognitive outcomes in sedentary older adults: the LIFE randomized trial. JAMA 2015;314(8):781-90.

13. Loprinzi PD, Scott T, Ikuta T, et al. Association Between Physical Activity on Changes in Cognitive Function: Boston Puerto Rican Health Study. Phys Sportsmed 2018;9:1-5. doi: 10.1080/00913847.2018.1547087

14. Sabia S, Dugravot A, Dartigues J-F, et al. Physical activity, cognitive decline, and risk of dementia: 28 year follow-up of Whitehall II cohort study. BMJ 2017;357:j2709.

15. Cheval B, Sieber S, Guessous I, et al. Effect of early-and adult-life socioeconomic circumstances on physical inactivity. Med Sci Sports Exerc 2018;50(3):476-85.

16. Daly M, McMinn D, Allan JL. A bidirectional relationship between physical activity and executive function in older adults. Front Hum Neurosci 2015;8:1044. 
17. Börsch-Supan A, Brandt M, Schröder M. SHARELIFE - One century of life histories in Europe. Adv Life Course Res 2013;18(1):1-4. doi: http://dx.doi.org/10.1016/j.alcr.2012.10.009

18. Lindwall M, Larsman P, Hagger MS. The reciprocal relationship between physical activity and depression in older European adults: a prospective cross-lagged panel design using SHARE data. Health Psychol 2011;30(4):453-62. doi: 10.1037/a0023268.

19. de Souto Barreto P, Cesari M, Andrieu S, et al. Physical Activity and Incident Chronic Diseases: A Longitudinal Observational Study in 16 European Countries. Am J Prev Med 2017;52(3):373-78. doi: 10.1016/j.amepre.2016.08.028

20. Harris S, Dowson J. Recall of a 10-word list in the assessment of dementia in the elderly. Br J Psychiatry 1982;141(5):524-27.

21. Rosen WG. Verbal fluency in aging and dementia. J Clin Exp Neuropsychol 1980;2(2):13546.

22. Aartsen MJ, Cheval B, Sieber S, et al. Advantaged socioeconomic conditions in childhood are associated with higher cognitive functioning but stronger cognitive decline in older age. PNAS 2019;116(12):5478-86.

23. Lezak MD, Howieson DB, Loring DW, et al. Neuropsychological assessment: Oxford University Press, USA 2004.

24. Zhao Q, Lv Y, Zhou Y, et al. Short-term delayed recall of auditory verbal learning test is equivalent to long-term delayed recall for identifying amnestic mild cognitive impairment. Plos One 2012;7(12):e51157.

25. Shao Z, Janse E, Visser K, et al. What do verbal fluency tasks measure? Predictors of verbal fluency performance in older adults. Front Psychol 2014;5:772.

26. United Nations Educational. International Standard Classification of Education 1997. UNESCO, Paris 2006

27. Cullati S, Kliegel M, Widmer E. Development of reserves over the life course and onset of vulnerability in later life. Nat Hum Behav 2018:1.

28. Boisgontier MP, Cheval B. The anova to mixed model transition. Neurosci Biobehav Rev 2016;68:1004-05.

29. Bates D, Mächler M, Bolker B, et al. Fitting Linear Mixed-Effects Models Using lme4. 2015 2015;67(1):48. doi: 10.18637/jss.v067.i01 [published Online First: 2015-10-07]

30. R Core Team. R: A language and environment for statistical computing. Vienna, Austria2017 [Available from: https://www.R-project.org/.

31. Kuznetsova A, Brockhoff, P.B., Christensen, R.H.B. ImerTest: Tests in Linear Mixed Effects Models. R package version 2.0-33. 2016 [Available from: https://CRAN.Rproject.org/package=lmerTest.

32. McArdle J. A latent difference score approach to longitudinal dynamic structural analyses. In: Cudeck R, du Toit S, Sorbom D, eds. Structural equation modeling: Present and future: Lincolnwood, IL: Scientific Software International. 2001:342-80.

33. Meredith W, Tisak J. Latent curve analysis. Psychometrika 1990;55(1):107-22.

34. Jöreskog KG. Estimation and testing of simplex models. ETS Research Bulletin Series 1970;1970(2):i-45.

35. Aichele S, Ghisletta P, Corley J, et al. Fluid Intelligence Predicts Change in Depressive Symptoms in Later Life: The Lothian Birth Cohort 1936. Psychol Sci 2018:1-12. doi: $10.1177 / 0956797618804501$

36. Aichele S, Ghisletta P, Neupert S. Memory Deficits Precede Increases in Depressive Symptoms in Later Adulthood. J Gerontol B Psychol Sci Soc Sci 2018

37. Grimm KJ, Ram N, Estabrook R. Growth modeling: Structural equation and multilevel modeling approaches. New York, NY: Guilford Press. 2016.

38. Muthén LK, Muthéne BO. Mplus user's guide (7th ed.). Los Angeles, CA2012. 
39. Wagenmakers E-J, Farrell S. AIC model selection using Akaike weights. Psychon Bull Rev 2004;11(1):192-96.

40. Cheval B, Radel R, Neva JL, et al. Behavioral and neural evidence of the rewarding value of exercise behaviors: a systematic review. Sports Med 2018;48(6):1389-404. doi: $10.1101 / 211425$

41. Cheval B, Tipura E, Burra N, et al. Avoiding sedentary behaviors requires more cortical resources than avoiding physical activity: An EEG study. Neuropsychologia 2018;119:68-80.

42. Cheval B, Sarrazin P, Isoard-Gautheur S, et al. Reflective and impulsive processes explain (in)effectiveness of messages promoting physical activity: a randomized controlled trial. Health Psychol 2015;34(1):10-19.

43. Cheval B, Sarrazin P, Boisgontier MP, et al. Temptations toward behaviors minimizing energetic costs (BMEC) automatically activate physical activity goals in successful exercisers. Psychol Sport Exerc 2017;30:110-17.

44. Prince SA, Adamo KB, Hamel ME, et al. A comparison of direct versus self-report measures for assessing physical activity in adults: a systematic review. Int J Behav Nutr Phys Act 2008;5(1):56.

45. Fitzpatrick S, Gilbert S, Serpell L. Systematic review: are overweight and obese individuals impaired on behavioural tasks of executive functioning? Neuropsychol Rev 2013;23(2):138-56.

46. Miyake A, Friedman NP. The nature and organization of individual differences in executive functions: Four general conclusions. Curr Dir Psychol Sci 2012;21(1):8-14.

47. Lee I-M, Shiroma EJ, Lobelo F, et al. Effect of physical inactivity on major noncommunicable diseases worldwide: an analysis of burden of disease and life expectancy. Lancet 2012;380(9838):219-29.

48. Nelson ME, Rejeski WJ, Blair SN, et al. Physical activity and public health in older adults: recommendation from the American College of Sports Medicine and the American Heart Association. Circulation 2007;116(9):1094. 
Table captions:

Table 1. Sample characteristics by baseline engagement in moderate physical activity. Physically active participants scored 1 or 2 on the item assessing moderate physical activity. Physically inactive participants scored 3 or $4 . \mathrm{SD}=$ standard deviation.

Table 2. Summary of the association of cognitive resources with the levels and trajectories of moderate physical activity across aging. Notes. All models were adjusted for gender, measurement occasion, dementia, chronic conditions, country of residence, birth cohort, and attrition. The models estimating the level of physical activity did not include interactions between cognitive resources and age (linear and quadratic). The models estimating the rate of change of physical activity included these interactions. Age (10y follow-up)" and "Age (10 y follow-up) squared" estimated the linear and quadratic changes in the odds of engagement in physical activity over a 10-year period.

Table 3. Summary of the changes in fit of the bivariate latent change score models. CFI = comparative fit index; chi2 = deviance $(-2 \times$ log-likelihood $)$; $\mathrm{df}=$ change in deviance per degrees freedom; $\mathrm{BIC}=$ Bayesian information criterion; $\mathrm{w}(\mathrm{BIC})$; RMSEA = root mean square error of approximation. Delta chi2 is estimated in comparison with the no coupling model, with lower values indicating better fi; $P=\mathrm{p}$-value for the log likelihood-ratio test of change in model fit. Models with a uni-directional coupling from vigorous physical activity to changes in verbal fluency did not converge. 
Table 1.

\begin{tabular}{|c|c|c|c|c|c|}
\hline \multirow[b]{3}{*}{ Cognitive resources } & \multicolumn{2}{|c|}{$\begin{array}{l}\text { Physically active } \\
\quad(n=18345)\end{array}$} & \multicolumn{2}{|c|}{$\begin{array}{c}\text { Physically } \\
\text { Inactive } \\
(\mathbf{n}=\mathbf{8 6 8 6 1})\end{array}$} & \multirow{2}{*}{$P$} \\
\hline & & & & & \\
\hline & & & & & \\
\hline Delayed recall (number of words), SD & 3.9 & 2.1 & 2.9 & 2.1 & $<.001$ \\
\hline Verbal fluency (number of words), SD & 20.4 & 7.4 & 16.0 & 7.8 & $<.001$ \\
\hline \multicolumn{6}{|l|}{ Education } \\
\hline Primary & 18172 & $20.9 \%$ & 6617 & $36.1 \%$ & \\
\hline Secondary & 48964 & $56.4 \%$ & 9240 & $50.4 \%$ & \\
\hline Tertiary & 19725 & $22.7 \%$ & 2488 & $13.5 \%$ & $<.001$ \\
\hline \multicolumn{6}{|l|}{ Covariates } \\
\hline Age at baseline (years), SD & 62.8 & 9.2 & 67.4 & 10.9 & $<.001$ \\
\hline \multicolumn{6}{|l|}{ Gender } \\
\hline Women & 47142 & $54.3 \%$ & 10726 & $58.5 \%$ & \\
\hline Men & 39719 & $45.7 \%$ & 7619 & $41.5 \%$ & $<.001$ \\
\hline \multicolumn{6}{|l|}{ Dementia } \\
\hline Yes & 1716 & $2.0 \%$ & 1083 & $5.9 \%$ & \\
\hline No & 85145 & $98.0 \%$ & 17262 & $94.1 \%$ & $<.001$ \\
\hline \multicolumn{6}{|l|}{ Chronic conditions ( 2 or more) } \\
\hline Yes & 34223 & $39.4 \%$ & 10320 & $56.3 \%$ & \\
\hline No & 52628 & $60.6 \%$ & 8025 & $43.7 \%$ & $<.001$ \\
\hline \multicolumn{6}{|l|}{ Countries } \\
\hline Belgium & 7073 & $8.1 \%$ & 1373 & $7.5 \%$ & \\
\hline Austria & 4675 & $5.4 \%$ & 942 & $5.1 \%$ & \\
\hline Denmark & 4760 & $5.5 \%$ & 422 & $2.3 \%$ & \\
\hline France & 5782 & $6.7 \%$ & 1195 & $6.5 \%$ & \\
\hline Germany & 6275 & $7.2 \%$ & 833 & $4.5 \%$ & \\
\hline Greece & 4678 & $5.4 \%$ & 1141 & $6.2 \%$ & \\
\hline Israel & 2220 & $2.6 \%$ & 699 & $3.8 \%$ & \\
\hline Italy & 5268 & $6.1 \%$ & 2097 & $11.4 \%$ & \\
\hline Netherlands & 4686 & $5.4 \%$ & 554 & $3.0 \%$ & \\
\hline Spain & 5985 & $6.9 \%$ & 1363 & $7.4 \%$ & \\
\hline Sweden & 5236 & $6.0 \%$ & 401 & $2.2 \%$ & \\
\hline Switzerland & 3602 & $4.1 \%$ & 488 & $2.7 \%$ & \\
\hline Czech Republic & 6386 & $7.4 \%$ & 1710 & $9.3 \%$ & \\
\hline Ireland & 827 & $0.9 \%$ & 139 & $0.8 \%$ & \\
\hline Poland & 1999 & $2.3 \%$ & 766 & $4.2 \%$ & \\
\hline Estonia & 5991 & $6.9 \%$ & 1325 & $7.2 \%$ & \\
\hline Hungary & 2161 & $2.5 \%$ & 729 & $4.0 \%$ & \\
\hline Portugal & 1275 & $1.5 \%$ & 740 & $4.0 \%$ & \\
\hline Slovenia & 4301 & $4.9 \%$ & 778 & $4.2 \%$ & \\
\hline Luxembourg & 1683 & $1.9 \%$ & 278 & $1.5 \%$ & \\
\hline Croatia & 1998 & $2.3 \%$ & 372 & $2.0 \%$ & $<.001$ \\
\hline \multicolumn{6}{|l|}{ Birth cohort } \\
\hline After 1945 & 50621 & $58.3 \%$ & 7701 & $42.0 \%$ & \\
\hline between 1939 and 1945 & 17085 & $19.7 \%$ & 3331 & $18.2 \%$ & \\
\hline between 1929 and 1938 & 14914 & $17.2 \%$ & 4746 & $25.9 \%$ & \\
\hline between 1919 and 1928 & 4241 & $4.8 \%$ & 2567 & $13.9 \%$ & $<.001$ \\
\hline
\end{tabular}


Table 2.

\begin{tabular}{|c|c|c|c|c|c|c|c|c|}
\hline \multirow[b]{2}{*}{ Moderate physical activity } & \multicolumn{2}{|c|}{$\begin{array}{c}\text { Models delayed recall } \\
\text { only }\end{array}$} & \multicolumn{2}{|c|}{$\begin{array}{l}\text { Models verbal fluency } \\
\text { only }\end{array}$} & \multicolumn{2}{|c|}{$\begin{array}{l}\text { Models education } \\
\text { only }\end{array}$} & \multicolumn{2}{|l|}{ Full Models } \\
\hline & $\mathrm{b}(95 \mathrm{CI})$ & $P$ & $b(95 \mathrm{CI})$ & $P$ & $b(95 \mathrm{CI})$ & $P$ & $b(95 \mathrm{CI})$ & $P$ \\
\hline \multicolumn{9}{|l|}{ Level } \\
\hline \multicolumn{9}{|l|}{ Cognitive resources } \\
\hline \multicolumn{9}{|l|}{ Between-person effects } \\
\hline Delayed recall & $0.06(0.06-0.06)$ & $<.001$ & & & & & $0.02(0.02-0.03)$ & $<.001$ \\
\hline Verbal fluency & & & $0.03(0.02-0.03)$ & $<.001$ & & & $0.02(0.02-0.02)$ & $<.001$ \\
\hline \multicolumn{9}{|l|}{ Education (ref. primary) } \\
\hline Secondary & & & & & $0.14(0.13-0.16)$ & $<.001$ & $0.08(0.06-0.09)$ & $<.001$ \\
\hline Tertiary & & & & & $0.21(0.20-0.23)$ & $<.001$ & $0.07(0.05-0.09)$ & $<.001$ \\
\hline \multicolumn{9}{|l|}{ Within-person effects } \\
\hline Delayed recall & $0.01(0.01-0.02)$ & $<.001$ & & & & & $0.01(0.01-0.01)$ & $<.001$ \\
\hline Verbal Fluency & & & $0.01(0.01-0.01)$ & $<.001$ & & & $0.01(0.01-0.01)$ & $<.001$ \\
\hline \multicolumn{9}{|l|}{ Rate of change (trajectories) } \\
\hline Age (10y follow-up) & $-0.22(-0.23-0.20)$ & $<.001$ & $-0.20(-0.22-0.19)$ & $<.001$ & $-0.25(-0.26-0.23)$ & $<.001$ & $-0.19(-0.20-0.17)$ & $<.001$ \\
\hline Age (10y follow-up) squared & $-0.11(-0.12-0.11)$ & $<.001$ & $-0.11(-0.11-0.10)$ & $<.001$ & $-0.12(-0.12-0.11)$ & $<.001$ & $-0.11(-0.11-0.10)$ & $<.001$ \\
\hline \multicolumn{9}{|l|}{ Cognitive resources } \\
\hline \multicolumn{9}{|l|}{ Between-person effects } \\
\hline Verbal fluency & & & $0.08(0.08-0.09)$ & $<.001$ & & & $0.07(0.06-0.08)$ & $<.001$ \\
\hline \multicolumn{9}{|l|}{ Education (ref. primary) } \\
\hline Secondary & & & & & $0.02(0.01-0.03)$ & .001 & $-0.01(-0.02-0.00)$ & .150 \\
\hline Tertiary & & & & & $0.08(0.07-0.10)$ & $<.001$ & $0.02(0.00-0.04)$ & .020 \\
\hline \multicolumn{9}{|l|}{ Non-linear (quadratic) effect } \\
\hline Delayed recall & $0.02(0.01-0.02)$ & $<.001$ & & & & & $0.00(0.00-0.01)$ & .210 \\
\hline Verbal Fluency & & & $0.02(0.02-0.03)$ & $<.001$ & & & $0.02(0.01-0.02)$ & $<.001$ \\
\hline \multicolumn{9}{|l|}{ Education (ref. primary) } \\
\hline Secondary & & & & & $0.03(0.02-0.04)$ & $<.001$ & $0.02(0.01-0.03)$ & $<.001$ \\
\hline Tertiary & & & & & $0.04(0.03-0.05)$ & $<.001$ & $0.03(0.01-0.04)$ & .001 \\
\hline \multicolumn{9}{|l|}{ Within-person effects } \\
\hline \multicolumn{9}{|l|}{ Linear effect } \\
\hline Delayed recall & $0.01(0.00-0.01)$ & .004 & & & & & $0.00(0.00-0.01)$ & 0.390 \\
\hline Verbal fluency & & & $0.02(0.01-0.02)$ & $<.001$ & & & $0.02(0.01-0.02)$ & $<.001$ \\
\hline \multicolumn{9}{|l|}{ Education (ref. primary) } \\
\hline \multicolumn{9}{|l|}{ Secondary } \\
\hline \multicolumn{9}{|l|}{ Tertiary } \\
\hline \multicolumn{9}{|l|}{ Non-linear (quadratic) effect } \\
\hline
\end{tabular}




\section{Table 3.}

\begin{tabular}{|c|c|c|c|c|c|c|c|c|c|c|}
\hline \multicolumn{11}{|l|}{ Moderate physical activity } \\
\hline Models & Parameters & chi2 & df & CFI & $\mathrm{BIC}$ & $\mathrm{w}(\mathrm{BIC})$ & RMSEA & delta chi2 & delta df & \\
\hline \multicolumn{11}{|l|}{ Delayed recall } \\
\hline No coupling & 19 & 1845.90 & 46 & .981 & 1772551.61 & .064 & .018 & & & \\
\hline Delayed recall $\rightarrow$ changes in moderate physical activity & 20 & 1828.97 & 45 & .982 & 1772546.52 & .816 & .018 & -16.93 & 1 & $<$ \\
\hline Moderate physical activity $\rightarrow$ changes in delayed recall & 20 & 1833.26 & 45 & .981 & 1772550.82 & .095 & .018 & -12.64 & 1 & $<$ \\
\hline Full coupling & 21 & 1824.22 & 44 & .982 & 1772553.47 & .025 & .018 & -21.68 & 2 & $<$ \\
\hline \multicolumn{11}{|l|}{ Verbal fluency } \\
\hline No coupling & 19 & 1431.12 & 46 & .988 & 2387128.17 & .235 & .016 & & & \\
\hline Verbal fluency $\rightarrow$ changes in moderate physical activity & 20 & 1417.17 & 45 & .988 & 2387125.91 & .729 & .016 & -13.95 & 1 & $<$ \\
\hline Moderate physical activity $\rightarrow$ changes in verbal fluency & 20 & 1423.34 & 45 & .988 & 2387132.08 & .033 & .016 & -7.78 & 1 & $<$ \\
\hline Full coupling & 21 & 1416.58 & 44 & .988 & 2387137.01 & .003 & .016 & -14.55 & 2 & $<$ \\
\hline
\end{tabular}

